\title{
$p$-ALGEBRAS OF EXPONENT $p$ *
}

\author{
BY NATHAN JACOBSON $\dagger$
}

A. A. Albert and O. Teichmüller have recently investigated the structure of $p$-algebras, that is, normal simple algebras of degree $p^{e}$ and characteristic $p . \ddagger$ In particular they showed that a necessary and sufficient condition that such an algebra have exponent $p$ is that it be similar to an algebra $A$ having a maximal subfield $C=F\left(c_{1}, c_{2}, \cdots, c_{m}\right)$, where $c_{i}{ }^{p}=\gamma_{i} \epsilon F$, the underlying field. The latter algebra is cyclic. It is the purpose of this note to apply some results of my paper Abstract derivation and Lie algebras $\S$ to obtain a new generation of $A$. For $m=1$ this generation is more symmetric than the cyclic generation. We obtain a condition that $A$ be a matrix algebra in terms of the new generation, and when $m=1$ we have as a consequence a reciprocity law for fields of characteristic $p$.

Let $A$ be a normal simple algebra of degree $p^{m}$ (order $p^{2 m}$ ) over a field $F$ of characteristic $p$ and suppose $A$ contains the maximal subfield $C=F\left(c_{1}, c_{2}, \cdots, c_{m}\right), c_{i}^{p}=\gamma_{i} \epsilon F$. Let $D$ be an arbitrary derivation of $C$ over $F$, that is, a mapping $x \rightarrow x D$ of $C$ into itself such that

$$
\begin{aligned}
(x+y) D & =x D+y D, & & (x \alpha) D=(x D) \alpha, \\
(x y) D & =(x D) y+x(y D), & & \alpha \in F .
\end{aligned}
$$

It is known that $D$ may be chosen so that the only elements $z$ such that $z D=0$ are those of $F, \|$ and for a $D$ of this type I have shown that

$$
x\left(D^{p^{m}}+D^{p^{m-1}} \tau_{1}+\cdots+D \tau_{m}\right)=0, \quad \tau_{i} \epsilon F,
$$

* Presented to the Society, April 10, 1937.

$\dagger$ National Research Fellow.

$\ddagger$ A. A. Albert, On normal division algebras of degree $p^{e}$ over $F$ of characteristic p, Transactions of this Society, vol. 39 (1936), pp. 183-188, and Simple algebras of degree pe over a centrum of characteristic $p$, Transactions of this Society, vol. 40 (1936), pp. 112-126. O. Teichmüller, p Algebren, Deutsche Mathematik, vol. 1 (1936), pp. 362-388.

$\S$ Transactions of this Society, vol. 42 (1937), pp. 206-224, referred to as J.

|| R. Baer, Algebraische Theorie der differentierbaren Funktionenkörper. I, Sitzungsberichte Heidelberger Akademie, 1927, pp. 15-32. 
or

$$
x^{\left(p^{m}\right)}+x^{\left(p^{m-1}\right)} \tau_{1}+\cdots+x^{\prime} \tau_{m}=0
$$

for all $x \epsilon C$, but no equation of the form

$$
x^{(r)}+x^{(r-1)} b_{1}+\cdots+x^{\prime} b_{r-1}+b_{r}=0, \quad b_{i} \epsilon,
$$

can hold if $r<p^{m}$. $^{*}$ I have shown also that any derivation in a simple subalgebra of a normal simple algebra may be extended to an inner derivation in the latter. $\dagger$ Thus there exists an element $d$ in $A$ such that $[x, d] \equiv x d-d x=x D$ for all $x \epsilon C$.

We note that

$$
x d^{k}=d^{k} x+C_{k, 1} d^{k-1} x^{\prime}+\cdots+x^{(k)},
$$

where the coefficients are those of the binomial theorem, and hence $x d^{p^{i}}=d^{p^{i}} x+x^{\left(p^{i}\right)}$. It follows from (1) that $\left(d^{p^{m}}+d^{p^{m-1}} \tau_{1}\right.$ $\left.+\cdots+d \tau_{m}\right)$ commutes with every $x$, and since $C$ is a maximal subfield of $A,\left(d^{p^{m}}+d^{p^{m-1}} \tau_{1}+\cdots+d \tau_{m}\right)=c \epsilon C$. Deriving with respect to $d$ (taking commutators), we have $[c, d]=0$, and so $c=\delta \epsilon F$ and

$$
d^{p^{m}}+d^{p^{m-1}} \tau_{1}+\cdots+d \tau_{m}=\delta .
$$

We assert that $C$ and $d$ generate the whole of $A$. Suppose

$$
d^{r}+d^{r-1} b_{1}+\cdots+b_{r}=0, \quad b_{i} \in C,
$$

is an equation of least degree having coefficients in $C$ and satisfied by $d$. If $x \in C$ by (2)

$$
d^{r-1} x_{1}+d^{r-2} x_{2}+\cdots+x_{r}=0,
$$

where, if we use the $C_{r, k}$ notation for binomial coefficients,

$$
\begin{gathered}
x_{1}=C_{r, 1} x^{\prime}, \quad x_{2}=C_{r, 2} x^{\prime \prime}+C_{r-1,1} x^{\prime} b_{1}, \cdots, \\
x_{r}=x^{(r)}+x^{(r-1)} b_{1}+\cdots+x^{\prime} b_{r-1} .
\end{gathered}
$$

Since (4) has minimum degree, $x_{1}=x_{2}=\cdots=x_{r}=0$. But by (1) $x_{r}=0$ is impossible for all $x$ unless $r \geqq p^{m}$. It follows that $r=p^{m}$ and $1, d, \cdots, d^{p^{m}-1}$ are (right) independent over $C$. Thus $C$ and $d$ generate an algebra of order $p^{m}$ over $C$ and hence $p^{2 m}$ over $F$, and so $C$ and $d$ generate all of $A$. The field $C$, the derivation

* J, p. 218.

$\dagger$ J, p. 214. 
$D$, and the equation (3) give a complete description of $A$.

Let $V(x)$ for $x \in C$ be the function

$$
V_{p^{m}}(x)+V_{p^{m-1}}(x) \tau_{1}+\cdots+V_{1}(x) \tau_{m},
$$

where

$$
V_{p^{j}}(x)=x^{p^{j}}+\left(x^{(p-1)}\right)^{p^{j-1}}+\left(x^{\left(p^{2}-1\right)}\right)^{p j-2}+\cdots+x^{\left(p^{j-1}\right)} .
$$

I have shown that $V(x) \epsilon F$, and that it has properties analogous to the norm in cyclic fields. * Now suppose $\delta=V\left(x_{0}\right)$. If $d_{1}=d-x_{0}$, then $\left[x, d_{1}\right]=x D$ for all $x$, and since

$$
d_{1}^{p^{j}}=\left(d-x_{0}\right)^{p^{j}}=d^{p^{j}}-V_{p^{j}}\left(x_{0}\right),
$$

we have

$$
d_{1}^{p^{m}}+d_{1}^{p^{m-1}} \tau_{1}+\cdots+d_{1} \tau_{m}=0,
$$

and so $A \cong F_{p^{m}}$, the algebra of all $p^{m}$-rowed square matrices with elements in $F$. $\dagger$

Conversely suppose that $A \cong F_{p^{m}}$. Then there exists in $A$ a field $\tilde{C} \cong C$ and an element $\tilde{d_{1}}$ such that $\left[\tilde{x}, \tilde{d}_{1}\right]=\widetilde{x D}$ where $x \longleftrightarrow \tilde{\sim}$ in the isomorphism between $C$ and $\tilde{C}$ and

$$
\tilde{d}_{1}^{p^{m}}+\tilde{d}_{1}^{p^{m-1}} \tau_{1}+\cdots+\tilde{d}_{1} \tau_{m}=0 .
$$

This isomorphism between $C$ and $\tilde{C}$ may be extended to an automorphism in $A$. Hence there exists an element $d_{1}$ corresponding to $\tilde{d}_{1}$ such that $\left[x, d_{1}\right]=x D$ and

$$
d_{1}^{p^{m}}+d_{1}^{p^{m-1}} \tau_{1}+\cdots+d_{1} \tau_{m}=0 .
$$

We observe that $d-d_{1}$ commutes with all the elements of $C$, and hence $d_{1}=d-x_{0}, x_{0} \epsilon C$. It follows as before that $\delta=V\left(x_{0}\right)$.

Theorem. $A$ necessary and sufficient condition that $A$ be $\cong F_{p^{m}}$ is that $\delta=V\left(x_{0}\right), x_{0} \in C$.

We now consider the special case where $m=1, C=F(c)$, $c^{p}=\gamma$. Let $D$ be the derivation such that $c D=1$. It is easily seen that $D^{p}=0$ and hence $A$ is generated by $c$ and $d$ such that

* See J, p. 224.

$\dagger$ The symbol $\cong$ denotes isomorphism. For the above equations and result see J, p. 223.

$\ddagger$ M. Deuring, Algebren, 1935, p. 42. 
$[c, d]=1$ and $d^{p}=\delta$. Thus $A$ has the basis $d^{i} c^{j}(i, j=0,1, \cdots$, $p-1)$ such that

$$
c^{p}=\gamma, \quad d^{p}=\delta, \quad c d-d c=1 .
$$

The condition that $A \cong F_{p}$ is $\delta=V\left(x_{0}\right), x_{0} \epsilon F(c)$. Here $V(x)=x^{p}$ $+x^{(p-1)}$, and so if $x=\xi_{0}+c \xi_{1}+\cdots+c^{p-1} \xi_{p-1}$, then

$$
V(x)=\left(\xi_{0}^{p}-\xi_{p-1}\right)+\gamma \xi_{1} p+\cdots+\gamma^{p-1} \xi_{p-1}^{p} .
$$

If $\delta$ is not a $p$-th power, (5) is essentially symmetric in $c$ and $d$. We define the derivation $d \rightarrow d E=-1$ in $F(d)$. Since $E^{p}=0$, the condition that $A \cong F_{p}$ is that

$$
\gamma=V\left(y_{0}\right)=y_{0} E^{p-1}+y_{0}^{p}, \quad y_{0} \epsilon F(d) .
$$

But if

$$
y=\eta_{0}+d \eta_{1}+\cdots+d^{p-1} \eta_{p-1},
$$

then

$$
V(y)=\left(\eta_{0}^{p}-\eta_{p-1}\right)+\delta \eta_{1}{ }^{p}+\cdots+\delta^{p-1} \eta_{p-1}^{p} .
$$

Thus we have the following reciprocity theorem for arbitrary fields of characteristic $p$.

THEOREM. If $\gamma$ and $\delta$ are not $p$-th powers in $F$, then $\left(\xi_{0}{ }^{p}-\xi_{p-1}\right)$ $+\gamma \xi_{1}{ }^{p}+\cdots+\gamma^{p-1} \xi_{p-1}^{p}=\delta$ is solvable for $\xi_{i} \epsilon F$ if and only if $\left(\eta_{0}{ }^{p}-\eta_{p-1}\right)+\delta \eta_{1}{ }^{p}+\cdots+\delta^{p-1} \eta_{p-1}^{p}=\gamma$ is solvable for $\eta_{i} \epsilon F$.

University of Chicago 